\title{
DINÂMICA TECNOLÓGICA E DA PRODUÇÃO AGROPECUÁRIA EM MINAS GERAIS ENTRE 1996 E $2006^{1}$
}

\author{
Adriano Provezano Gomes ${ }^{2}$ \\ José Luiz Alcantara Filho ${ }^{3}$ \\ Paulo Roberto Scalco ${ }^{4}$
}

Resumo: Este artigo teve como objetivo analisar o uso dos fatores de produção, bem como os impactos de mudanças tecnológicas no setor agropecuário de Minas Gerais, no período de 1996 a 2006. Para identificar em quais regiões houve progresso tecnológico, utilizou-se o índice de Malmquist de produtividade total dos fatores. A partir dessa análise, pôde-se verificar que $67 \%$ das microrregiões tiveram algum tipo de avanço tecnológico. Em seguida, foram analisadas as mudanças tecnológicas a fim de classificálas como poupadoras de capital ou de trabalho. Verificou-se que as regiões onde ocorreu progresso tecnológico são responsáveis por $84 \%$ do valor da produção agropecuária do estado. Outro ponto observado foi que houve significativa predominância de tecnologias poupadoras de mão de obra. Essa nova dinâmica na tecnologia agropecuária do estado de Minas Gerais teve impacto no uso dos fatores, reduzindo a elasticidade de produção do trabalho e aumentando a do capital.

Palavras-chave: Mudança tecnológica; agropecuária; uso de fatores.

1 Recebido em: 12/09/2013; Aceito em: 24/03/2014.

2 Doutor em Economia Aplicada pela Universidade Federal de Viçosa (UFV). Professor Associado do Departamento de Economia da Universidade Federal de Viçosa (UFV). E-mail: apgomes@ufv.br

3 Mestre em Economia pela Universidade Federal de Viçosa (UFV). Professor Assistente II - Universidade Federal Fluminense (UFF). E-mail: zezo_filho@yahoo.com.br

4 Doutorando em Economia Aplicada pela Universidade Federal de Viçosa (UFV). Professor Assistente II - Universidade Federal de Goiás (UFG). Faculdade de Administração, Ciências Contábeis e Ciências Econômicas - FACE. E-mail: pauloscalco@yahoo.com.br 
Abstract: This paper aims to analyze the use of factors of production, as well as the impacts of technological changes in the agricultural and husbandry sector of Minas Gerais, during the period of 1996-2006. It was used the Malmquist total factor productivity index in order to identify regions in which technological progress had occurred. Based on this analysis it can be seen that $67 \%$ of the microregions presented some kind of technological advancement. Then the technological changes were analyzed so that they could be classified as capital-saving or labor-saving. It was found that the regions in which technological progress had occurred are responsible for $84 \%$ of the agricultural and husbandry production in the state. Another point observed was the significant predominance of labor-saving technologies. This new dynamics in agricultural and husbandry technologies in the state of Minas Gerais has impacted the use of factors, reducing the production elasticity of labor and increasing the production elasticity of capital.

Keywords: Technological change, agriculture and husbandry, use of factors.

\section{Introdução}

A agropecuária sempre desempenhou papel de destaque na economia de Minas Gerais. Segundo dados da Fundação João Pinheiro, o PIB agropecuário mineiro em 2007 foi de R \$ 63,016 bilhões, representando $31 \%$ do PIB estadual. Além disso, o PIB do agronegócio mineiro representou, naquele ano, $11 \%$ do nacional, superior à participação do PIB total do estado em relação ao nacional, que foi de $9 \%$.

Apesar da importância do setor em todo o estado, o processo de desenvolvimento agrícola se deu de maneira disforme ao longo do tempo, gerando disparidades regionais e diferenças na alocação dos fatores de produção. Nesse contexto, o presente trabalho procura identificar em quais regiões de Minas Gerais ocorreu progresso tecnológico agrícola, além de tipificar as mudanças tecnológicas, se poupadoras de capital ou de trabalho. Em outras palavras, pretende-se identificar onde ocorreram as maiores mudanças tecnológicas e se elas afetaram o uso relativo dos fatores de produção. 
O uso de pacotes tecnológicos distintos pode ter sido influenciado por diversos fatores, tais como condições naturais, interesse dos empresários do setor, disponibilidade de crédito, demanda por produtos agrícolas, políticas públicas, entre outros. Contudo, diversos estudos sinalizam que, a partir da implantação dos pacotes tecnológicos provenientes da modernização da agricultura, vem ocorrendo um processo de substituição do trabalho pelo capital que, por sua vez, se reflete na estrutura produtiva agrícola e na escolha da tecnologia a ser utilizada.

A identificação dos locais onde estão ocorrendo mudanças tecnológicas e o tipo dessas mudanças é de fundamental importância no planejamento estratégico da produção agropecuária em Minas Gerais. É preciso saber onde estão ocorrendo as mudanças tecnológicas poupadoras de capital e as poupadoras de trabalho, objetivando traçar políticas públicas mais efetivas, diferenciadas por região.

\section{Metodologia}

O procedimento metodológico deste trabalho está dividido em três etapas. A primeira visa a identificar em quais microrregiões de Minas Gerais ocorreu progresso tecnológico, usando a decomposição do índice de Malmquist de produtividade total dos fatores. Na segunda etapa, é tipificada a mudança tecnológica a partir da classificação de Hicks para definir se tal mudança foi poupadora de trabalho ou de capital. Já o terceiro procedimento consiste na estimação das funções de produção, visando a mensurar os produtos marginais dos fatores e, assim, identificar o tipo de mudança tecnológica. A estimação das funções de produção é feita na forma funcional translog. 


\subsection{Obtenção da medida de mudança tecnológica: o índice de Malmquist}

Existindo dados de insumos e de produtos para mais de um período de tempo, pode-se calcular a mudança na produtividade total dos fatores ocorrida entre dois períodos. Uma das formas de fazer isso é através do índice de Malmquist. Este indicador pode ser decomposto em um índice de mudança na eficiência técnica e um índice de mudança tecnológica. Em outras palavras, a análise de eficiência/produtividade pode ser desmembrada em duas partes: mudança da distância em relação à fronteira tecnológica (mudança na eficiência) e mudança da fronteira tecnológica ao longo do tempo, ou seja, fruto do progresso tecnológico, sendo este o objeto de interesse do trabalho.

O cálculo do índice de Malmquist entre os períodos t e t+1 é embasado em quatro funções distância: $\mathrm{d}_{0}^{\mathrm{t}}\left(\mathrm{x}_{\mathrm{t}}, \mathrm{y}_{\mathrm{t}}\right), \mathrm{d}_{0}^{\mathrm{t}+1}\left(\mathrm{x}_{\mathrm{t}+1}, \mathrm{y}_{\mathrm{t}+1}\right), \mathrm{d}_{0}^{\mathrm{t}+1}\left(\mathrm{x}_{\mathrm{t}}, \mathrm{y}_{\mathrm{t}}\right)$ e $\mathrm{d}_{0}^{\mathrm{t}}\left(\mathrm{x}_{\mathrm{t}+1}, \mathrm{y}_{\mathrm{t}+1}\right)$. As duas primeiras indicam, respectivamente, o uso dos dados dos períodos $t$ e $t+1$. A terceira função distância utiliza os dados do período $t$ com a tecnologia existente no período $t+1$. Analogamente, a última função utiliza dados do período $\mathrm{t}+1$ e tecnologia do período $t$. O índice de mudança na produtividade de Malmquist com orientação produto, apresentado em Caves et al. (1982), pode ser definido como:

$$
M_{0}^{t, t+1}\left(y_{t+1}, x_{t+1}, y_{t}, x_{t}\right)=\left[\frac{d_{0}^{t+1}\left(x_{t+1}, y_{t+1}\right)}{d_{0}^{t}\left(x_{t}, y_{t}\right)}\right] x\left[\frac{d_{0}^{t}\left(x_{t+1}, y_{t+1}\right)}{d_{0}^{t+1}\left(x_{t+1}, y_{t+1}\right)} \times \frac{d_{0}^{t}\left(x_{t}, y_{t}\right)}{d_{0}^{t+1}\left(x_{t}, y_{t}\right)}\right]^{1 / 2}
$$

em que o primeiro termo do lado direito da equação mede a mudança de eficiência e o segundo termo, o progresso tecnológico. A Figura 1 ilustra essa definição, considerando um modelo com um insumo e um produto. 


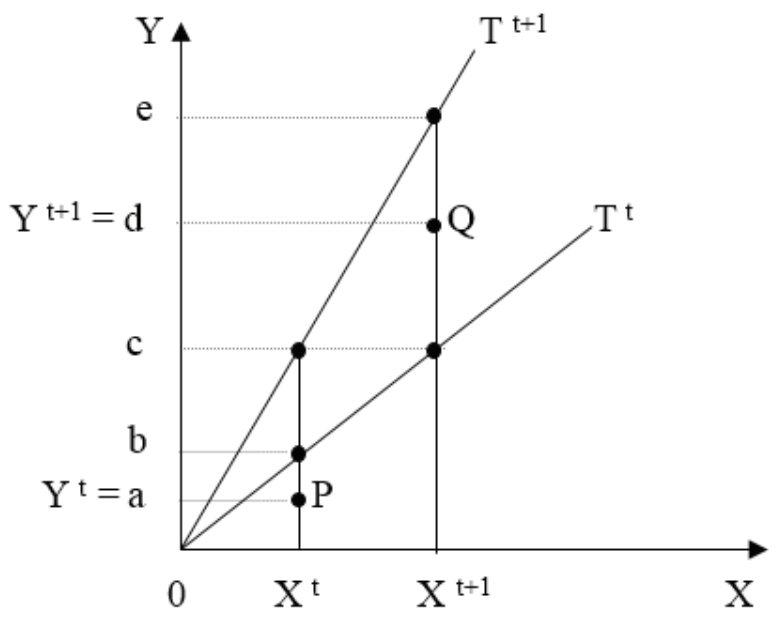

Figura 1 - Representação do índice de produtividade de Malmquist.

Na Figura 1, T e $\mathrm{T}^{\mathrm{t}+1}$ representam as tecnologias de produção em dois períodos. Considere uma unidade produtora (DMU) $)^{5}$ produzindo no ponto $\mathrm{P}$ no período $\mathrm{t}$ e no ponto $\mathrm{Q}$ no período $\mathrm{t}+1$. A mudança de eficiência para esta região é dada pela razão entre a eficiência técnica no período $\mathrm{t}+1$ em relação ao período $\mathrm{t}$, ou seja,

$$
\text { Mudança de eficiência }=\frac{0 d / 0 e}{0 a / 0 b}
$$

A mudança tecnológica é a média geométrica entre o deslocamento da tecnologia avaliada em $\mathrm{X}^{\mathrm{t}+1}$ e o deslocamento da tecnologia avaliada em $\mathrm{X}^{\mathrm{t}}$, obtida de seguinte forma:

$5 \mathrm{Na}$ literatura relacionada ao tema, uma unidade produtora é tratada como DMU (decision making unit), já que desses modelos provém uma medida para avaliar a eficiência relativa de unidades tomadoras de decisão. Por unidade produtora entende-se qualquer sistema produtivo que transforme insumos em produtos, podendo ser uma firma, um setor da economia ou uma região, como é o caso do presente trabalho 
Mudança tecnológica $=\left[\frac{0 \mathrm{~d} / 0 \mathrm{c}}{0 \mathrm{~d} / 0 \mathrm{e}} \times \frac{0 \mathrm{a} / 0 \mathrm{~b}}{0 \mathrm{a} / 0 \mathrm{c}}\right]^{1 / 2}$

Para calcular os componentes do índice, é necessário resolver quatro problemas de programação linear do tipo:

$\left[\mathrm{d}_{0}^{\mathrm{p}}\left(\mathrm{x}_{\mathrm{q}}, \mathrm{y}_{\mathrm{q}}\right)\right]^{-1}=\operatorname{MAX}_{\phi, \lambda} \quad \phi$, sujeito a :

$$
\begin{aligned}
& -\phi y_{i, q}+Y_{p} \lambda_{i} \geq 0, \\
& x_{i, q}-X_{p} \lambda_{i} \geq 0, \\
& \lambda_{1} \ldots \lambda_{n} \geq 0,
\end{aligned}
$$

em que $(\mathrm{p}, \mathrm{q}) \in\{(\mathrm{t}, \mathrm{t}),(\mathrm{t}+1, \mathrm{t}+1),(\mathrm{t}, \mathrm{t}+1),(\mathrm{t}+1, \mathrm{t})\}$.

Os valores obtidos para os $\phi$ 's indicam a quantidade máxima de aumento em todos os produtos do período q, com os insumos constantes requeridos para obter um ponto na função fronteira no período p. Conforme salientaram Marinho e Barreto (2000), para buscar evidências de qual ou quais DMUs podem estar deslocando a fronteira de produtividade, é necessário que sejam verificadas as três condições abaixo:

$$
\left[\frac{d_{0}^{t}\left(x_{t+1}, y_{t+1}\right)}{d_{0}^{t+1}\left(x_{t+1}, y_{t+1}\right)} \times \frac{d_{0}^{t}\left(x_{t}, y_{t}\right)}{d_{0}^{t+1}\left(x_{t}, y_{t}\right)}\right]^{1 / 2}>1
$$


$\mathrm{d}_{0}^{\mathrm{t}+1}\left(\mathrm{x}_{\mathrm{t}+1}, \mathrm{y}_{\mathrm{t}+1}\right)=1$

$\mathrm{d}_{0}^{\mathrm{t}}\left(\mathrm{x}_{\mathrm{t}+1}, \mathrm{y}_{\mathrm{t}+1}\right)>1$

A primeira condição reflete a presença de mudança tecnológica, descrita na segunda parte do lado direito da equação (1). A segunda condição indica que, se houver um deslocamento da fronteira, as DMU que o fazem devem estar situadas sobre ela. Por fim, a terceira condição afirma que, se o produto de uma DMU no período $t+1$ for superior ao máximo produto potencial que poderia ser obtido no período $t$, utilizando os fatores de produção do período $t+1$, é porque houve progresso tecnológico e a DMU pode estar deslocando a fronteira. Para mais detalhes sobre funções distância, veja Shepard (1970).

\subsection{Mudanças na função de produção e progresso tecnológico}

Após identificar em quais microrregiões de Minas Gerais houve progresso tecnológico, a próxima etapa consiste em verificar qual foi o tipo desta mudança tecnológica. Uma classificação muito utilizada nas análises econômicas se deve a Hicks (1932), que está relacionada às variações nas relações dos produtos marginais dos fatores. Para exemplificar essa classificação, considere os dois fatores de produção capital $(\mathrm{K})$ e trabalho (L), com seus respectivos produtos marginais $(P M g K$ e $P M g L)$. De acordo com Hicks, toda inovação deve aumentar a produtividade marginal de, pelo menos, um fator. Nesse sentido, as mudanças tecnológicas podem ser classificadas em:

- Poupadoras de trabalho: quando, para a combinação inicial de fatores, aumentar a relação $P M g K / P M g L$;

- Poupadoras de capital: quando, para a combinação inicial de fatores, diminuir a relação $P M g K / P M g L$; e

- Neutras: quando, para a combinação inicial de fatores, a relação $P M g$ K/ PMg Lse mantiver. 
A Figura 2 ilustra uma mudança tecnológica poupadora de trabalho. Considere que, inicialmente, a DMU esteja operando em equilíbrio no ponto A (isoquanta $I_{0}$ ), utilizando uma combinação de fatores $\mathrm{K}_{0}$ e $\mathrm{L}_{0}$ qualquer. Denominando os preços do capital e do trabalho de $\mathrm{P}_{\mathrm{K}}$ e $\mathrm{P}_{\mathrm{L}}$, respectivamente, o ponto de equilíbrio é alcançado quando

$$
\mathrm{P}_{\mathrm{K}} / \mathrm{P}_{\mathrm{L}}=\mathrm{PMg} \mathrm{K}_{0} / \mathrm{PMg} \mathrm{L}_{0}
$$

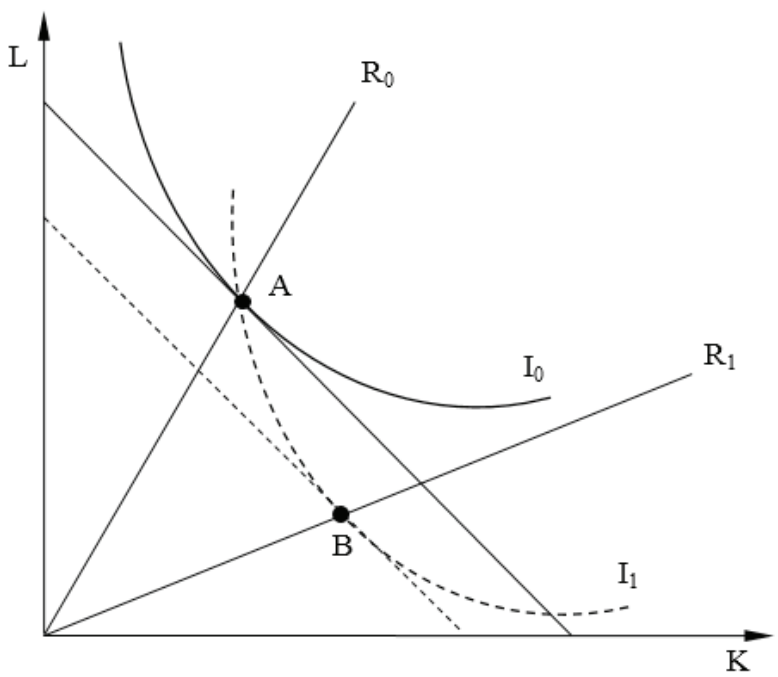

Figura 2 - Mudança tecnológica poupadora de trabalho.

Com a mudança tecnológica, a isoquanta de produção muda para $I_{1}$. Nesta nova isoquanta, mantendo-se a combinação inicial de fatores $\left(\mathrm{K}_{0} \mathrm{e} \mathrm{L}_{0}\right)$, os produtos marginais se alteram para $P M g K_{l}$ e $P M g L_{l}$. Considerando que a relação entre os preços dos fatores não se altera, a firma sairá do

ponto de equilíbrio, pois $\mathrm{PMg} \mathrm{K}_{1} / \mathrm{PMg} \mathrm{L}_{1}>\mathrm{PMg} \mathrm{K}_{0} / \mathrm{PMg} \mathrm{L}_{0}$. 
Para retornar ao equilíbrio, a firma terá que mudar sua combinação de fatores, de modo a restabelecer a proporcionalidade entre as produtividades marginais e os preços dos fatores. Para isso, é preciso diminuir a produtividade marginal do capital e aumentar a produtividade marginal do trabalho, ou seja, utilizar mais capital e menos trabalho. $\mathrm{O}$ novo ponto de equilíbrio ocorrerá em $\mathrm{B}$. Resumindo, inicialmente a firma estava operando ao longo do caminho de expansão $R_{0}$ e após a introdução da mudança tecnológica, o caminho de expansão se deslocou para $R_{1}$, onde se verifica maior relação capital/trabalho.

\subsection{Estimativa das funções de produção}

Conforme detalhado na seção anterior, para identificar o tipo de mudança tecnológica, é preciso conhecer os produtos marginais dos fatores em dois períodos de tempo. Para isso, é necessário estimar funções de produção. Existem várias formas funcionais que podem ser usadas para estimar a função de produção agrícola, tendo como variável dependente a produção (valor da produção) e, como explicativas, o pessoal ocupado (proxy para o fator trabalho) e o número de tratores (proxy para o fator capital).

Neste trabalho, optou-se por estimar as funções de produção na forma transcendental logarítmica (translog). A função de produção translog é considerada uma forma funcional flexível pelo fato de não impor restrições a priori aos valores das elasticidades de produção e de substituição entre os fatores. Além disso, a forma funcional translog pode representar de forma mais fiel a tecnologia de produção, possibilitando testes quanto à separabilidade, homogeneidade, monotonicidade, concavidade, entre outras características desejáveis para a tecnologia de produção.

Pressupondo-se separabilidade fraca entre os fatores de produção, de modo que eles possam ser agregados em dois grupos $\mathrm{X}_{1} \mathrm{e} \mathrm{X}_{2}$, a função de produção pode ser definida como: 
$\ln Y_{i}=\ln \alpha_{0}+\sum_{j=1}^{2} \alpha_{j} \ln X_{j i}+\frac{1}{2} \sum_{j=1}^{2} \sum_{k=1}^{2} \beta_{j k} \ln X_{j i} \ln X_{k i}+\mu_{i}$

em que $\mathrm{i}=1,2,3, \ldots, \mathrm{N}$ são as microrregiões; $\mathrm{X}_{\mathrm{jk}}, \mathrm{j}, \mathrm{k}=1,2$ são os fatores capital e trabalho; Y é a variável dependente (produção); $\alpha_{\mathrm{j}}$ e $\beta_{\mathrm{jk}}$ são os parâmetros a serem estimados; e $\mu_{\mathrm{i}}$ é um erro aleatório pressuposto normal, independente e de variância constante. A igualdade $\beta_{j k}=\beta_{k j}$ $, \mathrm{j}, \mathrm{k}=1,2$ é a condição imposta pelo teorema de Young, referente à igualdade das derivadas cruzadas de segunda ordem, garantindo as condições de simetria.

Estimando-se os parâmetros da função translog, podem ser calculados os produtos marginais (PMg) e as elasticidades de produção dos fatores $(\eta)$, utilizando as seguintes expressões:

$$
P M g X_{i}=\frac{\partial \ln Y}{\partial \ln X_{i}} \frac{Y}{X_{i}}=\left(\alpha_{i j}+\sum_{j} \beta_{i j} \ln X_{j}\right) \frac{Y}{X_{i}}
$$

$$
\eta X_{i}=\frac{\partial \ln Y}{\partial \ln X_{i}}=\alpha_{i j}+\sum_{j} \beta_{i j} \ln X_{j} .
$$

A monotonicidade é satisfeita quando os produtos marginais dos fatores forem positivos e a concavidade da função se verifica quando a matriz Hessiana orlada for negativa semidefinida. De acordo com Lima (2000), as condições de monotonicidade e concavidade podem não ser satisfeitas globalmente pela função translog, assim, tais propriedades devem ser testadas localmente (o ponto médio das variáveis é o mais usado). 


\subsection{Dados utilizados}

Para calcular o índice de Malmquist de produtividade total dos fatores e as funções de produção, foram utilizados dados de um produto e dois insumos. Os dados referem-se às 66 microrregiões do estado de Minas Gerais nos anos de 1996 e 2006. São eles:

- Valor da produção agropecuária (Y): soma do valor da produção animal e vegetal. Os dados da produção de lavouras foram obtidos na Pesquisa da Produção Agrícola Municipal e os da produção animal, na Pesquisa da Pecuária Municipal, ambas do IBGE. Para deflacionar os valores de 1996 para a base 2006, utilizou-se o Índice de Preços Recebidos pelos Produtores (IPR) da Fundação Getúlio Vargas.

- Número de tratores $\left(\mathrm{X}_{1}\right)$ : variável utilizada como proxy para o fator de produção capital. Os dados foram obtidos nos Censos Agropecuários do IBGE.

- Pessoal ocupado em estabelecimentos agropecuários: variável utilizada para representar o fator de produção Trabalho. Dados obtidos nos Censos Agropecuários do IBGE.

\section{Resultados e Discussão}

No período de 1996 a 2006, ocorreram significativas mudanças na composição do uso dos fatores de produção na agropecuária de Minas Gerais, conforme se pode observar na Tabela 1. Apesar de o número de estabelecimentos ter aumentado em cerca de $11 \%$, houve queda tanto na área utilizada quanto no uso de mão de obra, enquanto o número de tratores permaneceu praticamente inalterado. 
Tabela 1 - Número de estabelecimentos, área, pessoal ocupado e tratores utilizados na agropecuária de Minas Gerais, nos períodos de 1996 e 2006.

\begin{tabular}{ccccc}
\hline Especificação & Unidade & 1996 & 2006 & Variação (\%) \\
\hline Estabelecimento & Unidade & 496.677 & 550.529 & 10,84 \\
Área & Hectare & 40.811 .662 & 35.669 .793 & $-12,60$ \\
Mão de obra & Pessoa & 2.000 .046 & 1.860 .799 & $-6,96$ \\
Trator & Unidade & 89.667 & 89.789 & 0,14 \\
\hline
\end{tabular}

Fonte: IBGE - Censo Agropecuário.

Essa redução na relação mão de obra/trator ou, de forma genérica, trabalho/capital, fornece indícios de que as mudanças tecnológicas predominantes foram aquelas poupadoras de trabalho. Entretanto, não se pode afirmar que tais transformações ocorrem de forma homogênea em todas as regiões do estado. Diversos fatores podem ser responsáveis pelas diferenças intra e inter-regionais nas funções de produção agrícolas, principalmente em um estado com a dimensão territorial de Minas Gerais. O fato é que nas regiões onde as mudanças tecnológicas foram mais intensas, certamente houve alterações no uso dos fatores de produção.

Para analisar essa questão, os resultados apresentados a seguir serão divididos em três seções: inicialmente, serão identificadas as microrregiões de Minas Gerais onde ocorreram mudanças tecnológicas; depois, as mudanças tecnológicas serão classificadas em poupadoras de capital ou trabalho; e, por fim, procurar-se-á verificar o que aconteceu com os fatores de produção nas regiões onde ocorreram as mudanças tecnológicas. 


\subsection{Mudanças tecnológicas na agropecuária de Minas Gerais}

Para identificar onde ocorreram mudanças tecnológicas, utilizou-se o Índice de Malmquist de produtividade total dos fatores. A utilização desse índice é útil por permitir decompor a mudança na produtividade total dos fatores em mudança na eficiência técnica e mudança tecnológica.

Os resultados indicam que, das 66 microrregiões de Minas Gerais, em 44 delas houve algum tipo de ganho tecnológico na produção agrícola. Por outro lado, em 22 microrregiões, houve regresso tecnológico. Valores acima de uma unidade indicam regiões onde houve progresso tecnológico, ou seja, houve deslocamento para cima da fronteira de produção. Por outro lado, valores abaixo da unidade indicam retrocesso tecnológico. Quando o índice de mudança tecnológica for igual a um, significa que a tecnologia não se alterou entre os dois momentos de tempo analisados. A distribuição das microrregiões segundo as medidas de mudança tecnológica pode ser observada na Figura 3.

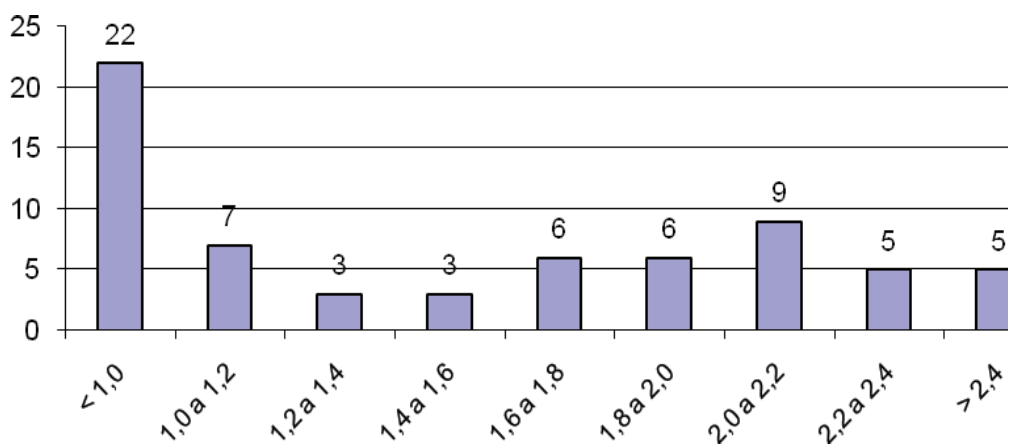

Figura 3 - Distribuição das microrregiões de Minas Gerais, segundo intervalos do índice de mudança tecnológica, no período de 1996 a 2006.

Como pode se verificar, das 44 microrregiões que apresentaram progresso tecnológico, em 19 delas tal progresso foi superior a $100 \%$ (índice acima de 2,0), o que representa um avanço tecnológico superior 
a 7,18\% ao ano. Esse número por si só mostra a magnitude do processo de mudança que ocorreu na agropecuária em várias regiões de Minas Gerais, porém de forma heterogênea. $\mathrm{O}$ que se verificou foi regresso tecnológico em um terço das microrregiões do estado. A existência de regresso tecnológico implica dizer que melhorias na produtividade total dos fatores somente serão possíveis se os ganhos em eficiência técnica forem proporcionalmente maiores.

Para identificar onde ocorreram as mudanças tecnológicas, na Figura 4 encontra-se a distribuição do índice no mapa de Minas Gerais, cujos valores foram separados em três estratos: até 1, o que corresponde a regiões onde houve regresso tecnológico; de 1 a 2, representando mudanças positivas porém inferiores a 7,18\% ao ano; e acima de 2 , representando as regiões onde o ganho tecnológico foi superior a 7,18\% ao ano.

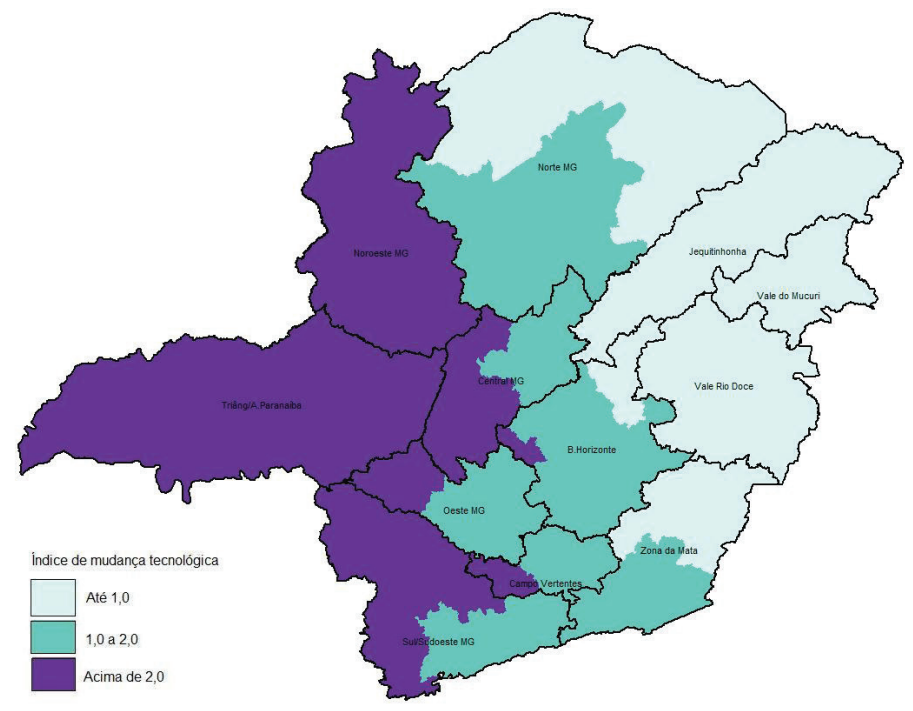

Figura 4 - Distribuição do índice de mudança tecnologia na agropecuária de Minas Gerais, no período de 1996 a 2006. 
Conforme se pode verificar, a divisão em estratos definiu três faixas bem delimitadas no mapa mineiro. Os locais onde os ganhos tecnológicos foram mais intensos são formados pelas mesorregiões Triângulo Mineiro/ Alto Paranaíba, Noroeste, além de parte do Sul de Minas, Oeste e Central Mineira. Por outro lado, entre as regiões onde não houve progresso tecnológico, encontram-se as mesorregiões do Jequitinhonha, Vale do Mucuri e Vale do Rio Doce, locais onde a agropecuária é menos pujante.

De fato, os dados apresentados na Tabela 2 confirmam a relação positiva entre mudança tecnológica e valor da produção, ou seja, as regiões onde houve maior mudança tecnológica são aquelas onde se concentra a maior parte da produção agropecuária.

Tabela 2 - Distribuição do valor da produção agropecuária de Minas Gerais, segundo estratos de mudança tecnológica. Dados de 2006.

\begin{tabular}{ccc}
\hline \multirow{2}{*}{$\begin{array}{c}\text { Estratos de mudança } \\
\text { tecnológica }\end{array}$} & \multicolumn{2}{c}{ Valor da produção agropecuária* } \\
\cline { 2 - 3 } & $\mathrm{R} \$$ mil & $\%$ \\
\hline Até 1 & 2.590 .669 & 16,03 \\
1 a 2 & 3.892 .059 & 24,08 \\
Acima de & 9.677 .433 & 59,88 \\
Total & 16.160 .161 & 100,00 \\
\hline
\end{tabular}

Fonte: IBGE - Produção Agrícola Municipal e Pesquisa da Pecuária Municipal.

De acordo com os dados, as 22 microrregiões que apresentaram regresso tecnológico representam apenas $16 \%$ do valor da produção agropecuária estadual. No outro extremo, as 19 microrregiões onde a mudança tecnológica foi maior concentram cerca de $60 \%$ do valor da produção.

A forma como o índice de mudança tecnológica foi calculado, por meio de funções distância, permite identificar quais regiões podem ser as responsáveis pelo deslocamento da fronteira de produção agrícola do estado. Apesar de 44 microrregiões terem apresentado ganho tecnológico, 
isto é, terem respeitado a condição descrita na equação (5), apenas a microrregião de Uberaba foi a responsável pelo deslocamento da fronteira. As demais não atenderam a uma das condições necessárias para o deslocamento da fronteira. Isso significa que ou não alcançaram máxima eficiência no ano de 2006 - condição descrita na equação (6) ou seu produto em 2006 não foi superior ao máximo produto potencial que poderia ser obtido no ano de 1996, utilizando os fatores de produção do ano de 2006 - condição descrita na equação (7). Com isso, mesmo havendo progresso tecnológico em várias regiões do estado, quem deslocou a fronteira foi a microrregião de Uberaba. As demais utilizaram essa nova fronteira como referência. De fato, Uberaba foi a microrregião que registrou o maior índice de mudança tecnológica, da ordem de 14,5\% ao ano, cerca de três vezes superior à média do estado.

\subsection{Tipificação das mudanças tecnológicas na agropecuária de Minas} Gerais

Após identificar onde ocorreram as mudanças tecnológicas, o passo seguinte foi verificar o tipo de mudança tecnológica, ou seja, se foi poupadora de trabalho ou de capital. Para isso, foi preciso estimar duas funções de produção, uma com dados de 1996 e outra, com dados de 2006.A forma funcional utilizada nas funções de produção foi a translog, conforme descrita em (8). Os parâmetros estimados encontram-se na Tabela 3 . 
Tabela 3 - Parâmetros estimados para as funções de produção agrícola de Minas Gerais, no período de 1996 e 2006.

\begin{tabular}{ccc}
\hline \multirow{2}{*}{ Variável } & \multicolumn{2}{c}{ Parâmetros estimados } \\
\cline { 2 - 3 } & 1996 & 2006 \\
\hline Intercepto & $17,9471^{*}$ & $18,3320^{*}$ \\
$\ln \mathrm{K}$ & $1,2321^{*}$ & $1,5461 *$ \\
$\ln \mathrm{L}$ & $-3,2037 *$ & $-3,5789 *$ \\
$1 / 2(\ln \mathrm{K})^{2}$ & $0,3279 * * *$ & $0,5966^{* * *}$ \\
$1 / 2(\ln \mathrm{L})^{2}$ & $0,5541^{*}$ & $0,7118^{* *}$ \\
$\ln \mathrm{K} * \ln \mathrm{L}$ & $-0,2722^{* *}$ & $-0,4610^{* * *}$ \\
$\mathrm{R}^{2}$ & 0,8007 & 0,8101 \\
\hline
\end{tabular}

Fonte: Dados da pesquisa.

$\mathrm{K}=$ capital (trator) e $\mathrm{L}=$ trabalho (mão de obra)

${ }^{*}$ Significativo a $10 \%$

${ }^{* *}$ Significativo a $5 \%$

$* * *$ significativos a $1 \%$.

Como se pode verificar, todos os coeficientes estimados para os dois anos foram significativos. Esses parâmetros foram utilizados para calcular os produtos marginais e as elasticidades de produção. Por exemplo, no ano de 2006, o valor médio da produção agrícola nas microrregiões mineiras foi de cerca de R $\$ 245$ milhões, sendo utilizados, em média, 28 mil pessoas e 1,3 mil tratores. Considerando tais dados e os parâmetros estimados na Tabela 3, são obtidos os valores médios dos produtos marginais e das elasticidades de produção, utilizando as equações (9) e (10), respectivamente.

$\mathrm{Na}$ Tabela 4 encontram-se os valores dos produtos marginais (PMg) e as elasticidades de produção do capital (trator) e do trabalho (mão de obra), calculados no ponto médio das variáveis, utilizando-se os parâmetros estimados da tabela anterior. 
Tabela 4 - Produtos marginais e elasticidades de produção do capital e do trabalho calculados no ponto médio das variáveis, no período de 1996 e 2006.

\begin{tabular}{ccc}
\hline Especificação & 1996 & 2006 \\
Elasticidade de produção K & 0,7889 & 1,1266 \\
Elasticidade de produção L & 0,5506 & 0,3888 \\
Produto marginal K & 75,2875 & 151,3850 \\
Produto marginal L & 2,3559 & 2,5212 \\
PMg K / PMg L & 31,9570 & 60,0448 \\
\hline
\end{tabular}

Fonte: Dados da pesquisa.

$\mathrm{K}=$ capital (trator) e $\mathrm{L}=$ trabalho (mão de obra)

Todas as elasticidades de produção dos fatores calculadas no ponto médio da amostra foram positivas, satisfazendo a condição de monotonicidade nas funções estimadas. De modo geral, o que se verifica é que a influência da mecanização no processo produtivo é maior que a da mão de obra nos dois períodos analisados. Entretanto, dois pontos chamam a atenção: primeiro, nota-se que a influência do capital aumentou, enquanto a do trabalho diminuiu. Com isso, a influência relativa do capital sobre o trabalho na produção agropecuária mineira aumentou. $\mathrm{O}$ segundo ponto é que, embora a elasticidade de produção do trabalho tenha diminuído, a elasticidade de escala aumentou de 1,3395 para 1,5154, graças ao aumento mais que proporcional na elasticidade de produção do capital. Pode-se dizer, então, que há forte evidência de que a produção agropecuária de Minas Gerais apresenta rendimentos crescentes de escala.

Em relação aos produtos marginais, nota-se que ambos aumentaram, embora o aumento no produto marginal do capital tenha sido significativamente superior ao do trabalho. Com isso, a relação $\mathrm{PMg}$ $\mathrm{K}$ / PMg L aumentou, indicando que, na média do estado, a tecnologia agropecuária poupou trabalho relativamente ao capital.

Para evidenciar o tipo de mudança tecnológica ocorrida em cada microrregião, utilizou-se da classificação proposta por Hicks, segundo a qual uma mudança tecnológica não neutra requer que a produtividade 
marginal de um fator de produção aumente em relação à do outro fator. Nesse sentido, a classificação da mudança tecnológica, se poupadora de capital ou de trabalho, dependerá da variação na relação entre os produtos marginais dos fatores. Caso a relação entre o produto marginal do capital e o produto marginal do trabalho aumente, a tecnologia é dita poupadora de trabalho. Caso contrário, será poupadora de capital.

Para identificar mudanças na taxa marginal de substituição técnica (razão entre os produtos marginais), os cálculos dos produtos marginais devem ser feitos considerando o mesmo conjunto inicial de dados, mudando apenas os coeficientes estimados. Em outras palavras, consideram-se os dados de 1996 para estimar os produtos marginais dos fatores em 1996 e em 2006, porém os parâmetros referem-se às funções de produção estimadas para cada ano, descritos na Tabela 3. Os resultados dos tipos de mudanças tecnológicas ocorridas nas microrregiões são mostrados na Tabela 5.

Tabela 5 - Tipos de mudança tecnológica ocorridos nas microrregiões de Minas Gerais que apresentaram progresso tecnológico na produção agropecuária, no período de 1996 a 2006.

\begin{tabular}{ccc}
\hline \multirow{2}{*}{ Tipo de mudança tecnológica } & \multicolumn{2}{c}{ Microrregiões } \\
\cline { 2 - 3 } & Número & $\%$ \\
\hline Poupadora de K & 7 & 15,91 \\
Poupadora de L & 37 & 84,09 \\
Total & 44 & 100,00 \\
\hline
\end{tabular}

Fonte: Dados da pesquisa.

Após identificar o tipo de mudança tecnológica ocorrida, verificou-se que, em $84 \%$ das microrregiões que apresentaram progresso tecnológico, a tecnologia foi poupadora de trabalho. Somente em 7 microrregiões isso não ocorreu. $\mathrm{O}$ fato de a tecnologia poupadora de trabalho ter predominado não significa necessariamente dizer que o uso de tratores foi intensificado, mas sim que o uso de tratores relativamente à mão de obra aumentou. Essa discussão será apresentada a seguir. 


\subsection{Impactos das mudanças tecnológicas no uso dos fatores de produção}

É de se esperar que a influência do capital no processo produtivo cresça em regiões onde a mudança tecnológica foi poupadora de trabalho. $\mathrm{O}$ contrário deve ocorrer em regiões onde não houve progresso técnico ou se houve mudança poupadora de capital. Os dados apresentados na Tabela 6 comprovam essa hipótese.

Tabela 6 - Médias das elasticidades de produção dos fatores nas microrregiões de Minas Gerais separadas pela ocorrência ou não de progresso tecnológico entre 1996 e 2006.

\begin{tabular}{llrr}
\hline \multirow{2}{*}{ Período } & \multirow{2}{*}{ Especificação } & \multicolumn{2}{c}{ Progresso tecnológico } \\
\cline { 3 - 4 } & & \multicolumn{1}{c}{ Sim } & Não \\
\hline \multirow{2}{*}{1996} & Elasticidade de produção K & 0,8486 & 0,2919 \\
& Elasticidade de produção L & 0,4174 & 0,9916 \\
\hline \multirow{2}{*}{2006} & Elasticidade de produção K & 1,2646 & 0,2161 \\
& Elasticidade de produção L & 0,1521 & 1,1252 \\
\hline \multirow{2}{*}{ Variação } & Elasticidade de produção K & $49,02 \%$ & $-25,96 \%$ \\
& Elasticidade de produção L & $-63,56 \%$ & $13,47 \%$ \\
\hline
\end{tabular}

Fonte: Dados da pesquisa.

Observando esses dados, dois pontos chamam a atenção. Em primeiro lugar, ao analisar um período isoladamente (1996 ou 2006), percebe-se que nas regiões onde houve progresso tecnológico a elasticidade de produção do capital é maior que a do trabalho. O inverso ocorre nas regiões que não apresentaram progresso na tecnologia. Isso significa que, para ocorrer um determinado aumento percentual na produção, o aumento no uso de tratores é mais importante onde há mudança tecnológica positiva, enquanto a intensificação no uso de mão de obra traz mais resultados nos locais onde não houve progresso tecnológico.

Nos locais onde houve progresso tecnológico, a elasticidade de produção do capital aumentou, e a elasticidade de produção do trabalho diminuiu. 
Já nas outras regiões, ocorreu o contrário. Isso significa que o capital ficou mais importante no processo produtivo em algumas regiões, enquanto o trabalho ficou mais importante em outras. Nesse ponto, deve-se destacar a importância de políticas agrícolas diferenciadas entre as regiões, visando ao aumento da produção agropecuária global do estado. Em outras palavras, caso se deseje aumentar a produção agrícola em todo o estado, em alguns locais tal aumento será proporcionalmente maior pela incorporação de tratores, enquanto em outros o melhor talvez seja manter o uso mais intensivo de mão de obra.

Uma vez que o progresso tecnológico ocorreu em determinadas regiões do estado, pode-se identificar seu impacto no uso relativo dos fatores de produção. Essa análise é feita utilizando os dados da Tabela 7.

Tabela 7 - Mudanças no uso relativo dos fatores de produção nas regiões onde ocorreu progresso tecnológico agropecuário, no período de 1996 a 2006.

\begin{tabular}{llccc}
\hline Especificação & Unidade & 1996 & 2006 & Variação (\%) \\
\hline Área & Hectare (ha) & 26.573 .344 & 24.927 .144 & $-6,19$ \\
Mão de obra & Pessoa & 26.266 & 23.840 & $-9,24$ \\
Trator & Unidade & 1.828 & 1.830 & 0,11 \\
Trator/área & Unidade/1000 ha & 0,0688 & 0,0734 & 6,72 \\
Mão de obra/área & Pessoa/1000 hectares & 0,9884 & 0,9564 & $-3,24$ \\
Mão de obra/trator & Pessoa/Unidade & 14,37 & 13,03 & $-9,34$ \\
\hline
\end{tabular}

Fonte: IBGE - Censos Agropecuários

Conforme discutido, entre 1996 e 2006, a produção agropecuária de Minas Gerais foi marcada pela redução no uso de terra e de mão de obra. Contudo, nas regiões onde houve progresso tecnológico, a redução no uso da terra foi inferior à média estadual, enquanto o corte de pessoal ocupado na agropecuária foi maior. Para o estado como um todo, a área agrícola se reduziu em $12,6 \%$ e a mão de obra, em 6,96\%. Já nas regiões com progresso tecnológico positivo, as reduções foram de $6,19 \%$ e $9,24 \%$, respectivamente. 
Uma vez que não houve alteração significativa no número de tratores, o que se verifica é uma ampliação na relação trator/área e uma redução também na relação mão de obra/trator. Nesse sentido, a introdução de tecnologias poupadoras de mão de obra não ocorreu pelo aumento no número de tratores, mas sim pela redução proporcionalmente maior no uso dos fatores terra e trabalho.

Por fim, pode-se verificar como a mudança tecnológica pode ter afetado o uso da terra destinada à produção agropecuária. É importante ressaltar que as mudanças apresentadas na Tabela 8 não devem ser atribuídas exclusivamente ao tipo de progresso tecnológico ocorrido em Minas Gerais. Outros fatores podem ter influenciado na tomada de decisão dos produtores na definição de qual atividade agropecuária desenvolver.

Tabela 8 - Distribuição do uso da terra na produção agropecuária das regiões de Minas Gerais onde ocorreu progresso tecnológico agropecuário, nos períodos de 1996 e 2006. Dados em hectares.

\begin{tabular}{lccc}
\hline Especificação & 1996 & 2006 & Variação (\%) \\
\hline Lavouras & 4.093 .185 & 5.051 .174 & 23,40 \\
Pastagens & 18.005 .753 & 14.773 .026 & $-17,95$ \\
Matas e florestas & 4.474 .406 & 5.102 .944 & 14,05 \\
Total & 26.573 .344 & 24.927 .144 & $-6,19$ \\
\hline
\end{tabular}

Fonte: IBGE - Censos Agropecuários

Apesar de outros fatores exercerem influência, o fato é que nas regiões onde houve progresso tecnológico a distribuição das terras teve significativa mudança. Conforme se pode observar, as áreas destinadas às lavouras e às matas e florestas tiveram aumentos significativos, enquanto a área de pastagem se reduziu também de forma significativa.

Segundo análises do IBGE, a substituição das áreas de pastagem por lavouras no período de 1996 a 2006 ocorreu em razão da progressiva 
inserção do país no mercado mundial de produção de grãos (especialmente a soja) e da intensificação da pecuária.

Outra possível explicação para a redução das pastagens é o abandono de áreas degradadas, fato comum e preocupante em diversas regiões do estado. Já o aumento nas áreas de matas e florestas ocorreu, basicamente, pela expansão de matas plantadas, com destaque para o cultivo de eucalipto.

\section{Conclusões}

O trabalho objetivou identificar em quais regiões de Minas Gerais ocorreu mudança tecnológica na agropecuária e qual o tipo dessa mudança. As microrregiões que apresentaram maiores índices de avanço tecnológico estão localizadas na região oeste do estado, onde ocorre produção de larga escala, geralmente voltada à exportação, e de agricultura integrada com o setor industrial, de uso intensivo em tecnologia e mecanização.

Em contraste, na região leste, estão as microrregiões que apresentaram retrocesso tecnológico, compreendendo as mesorregiões do Vale do Rio Doce, Jequitinhonha e Mucuri, regiões mais pobres, com menor participação no produto agropecuário do estado.

As consequências dessa diferença estadual da mudança tecnológica podem ser interpretadas sob duas óticas. A primeira é que a absorção de novas tecnologias gerou externalidades positivas à economia, uma vez que impulsionou o crescimento do setor nas regiões mais dinâmicas, onde a produção agropecuária é forte; a segunda ótica diz respeito à ampliação da dualidade tecnológica na agropecuária do estado. Nas regiões mais pobres do estado, onde a parcela da produção agrícola é pequena, não houve progresso tecnológico, ao contrário, predominaram as microrregiões onde se constatou retrocesso na tecnologia de produção agrícola. 
Com isso, pode-se dizer que a expansão no uso de capital proporcionou redução da mão de obra empregada na produção agropecuária, uma vez que o aumento da produção não ocorreu pela expansão no uso dos fatores, mas pelo aumento da produtividade do trabalho, sem que houvesse ocorrido incrementos no uso de tratores e de terra.

Outro ponto relevante é que, embora cerca de $82 \%$ da população mineira seja urbana e o estado se destaque no cenário industrial brasileiro, ainda existem regiões muito pobres, com baixa dinamização econômica e, predominantemente, rurais. Analisando os dados, percebe-se que o progresso tecnológico não chegou, ou foi em menor intensidade, a essas regiões. Contudo, a tendência de disseminação da tecnologia poupadora de mão de obra pode gerar condições de precariedade e fragilidade econômica ainda maiores.

Isso pode ocorrer, pois o progresso tecnológico sob a lógica poupadora de mão de obra, embora tenha propiciado aumento da produção, é conflitante com a geração de emprego. Resta saber se os outros setores da economia mineira são capazes de absorver a mão de obra que está deixando de ser utilizada no meio rural.

Deste modo, é preciso considerar que as alternativas para o crescimento da produção agropecuária devem ser diferentes entre as regiões do estado de Minas Gerais, levando-se em conta o impacto regional na distribuição da renda e emprego. Em outras palavras, é provável que políticas agrícolas diferenciadas entre as regiões sejam mais efetivas do que uma política global de crescimento da produção. 


\section{Referências}

CAVES, D.W, CHRISTENSEN, L.R., DIEWERT, W.E. The economic theory of index numbers and the measurement of Inputs, output, and productivity. Econometrica, 50(6), p. 1393-1414, 1982.

HICKS, J. R. The Theory of Wages. London: Macmillan, 1932. 261 p.

LIMA, J.E. Definições e aplicações de elasticidades de substituição: revisão e aplicação. Revista de Economia e Sociologia Rural. 38, $\mathrm{n}^{\circ} 1$, Jan/Mar 2000. P. 9-44.

MARINHO, E.L.L., BARRETO, F.A.F.D. Análise da produtividade e progresso tecnológico dos estados do Nordeste. Fortaleza: CENER, 2000. $30 \mathrm{p}$.

SHEPHARD, R.W. Theory of Cost and Production Functions. Princeton: Princeton University Press, 1970. 
REVISTA DE ECONOMIA E AGRONEGÓCIO, VOL.11, $N^{\circ} 2$ 